\title{
Three-Dimensional Computed Tomography Studies of the Tendons of the Foot and Ankle
}

\author{
R.J. Bellon and S.M. Horwitz
}

\begin{abstract}
Three dimensional (3D) reconstruction techniques were applied to serial computed tomography scans of a cadaveric foot. Subsequent manipulations of the image data on a 3D imaging workstation facilitated differentiation of tendon from surrounding tissue. Through the use of free-rotation and density thresholding, detailed 3D images of the major tendons were produced and displayed. The value of the technique for education and its potential for diagnosis is discussed. Copyright $\odot 1992$ by W.B. Saunders Company
\end{abstract}

KEY WORDS: computed tomography, three dimensional imaging, three dimensional reconstruction, image rendering, tendons, foot and ankle.

$\mathbf{F}$ OR SOME time the ability to reconstruct three dimensional (3D) images from "stacked" serial computed tomography (CT) slices has been available. Interactive 3D display systems show great promise as tools for the visualization of complex anatomy and pathology in both teaching and clinical environments. ${ }^{1}$

Bone is most easily isolated on these types of reconstructions because of its unique high density among human tissues. As a result, the clinical utility of 3D reconstruction has been demonstrated primarily in situations involving bony structures-eg, acetabular fractures ${ }^{2,3}$ and craniofacial surgical planning. ${ }^{4}$ Although some notable 3D reconstructions have been done on the lungs, ${ }^{5,6}$ most attempts to isolate soft tissues have been hampered by their lack of unique density ranges.

Our goal here was to further explore 3D soft tissue reconstructions, and in particular the tendons of the foot and ankle.

From the Department of Radiology, MetroHealth Medical Center/Case Westem Reserve University, Cleveland, $\mathrm{OH}$.

Supported by the Department of Radiology, MetroHealth Medical Center, Cleveland, $\mathrm{OH}$.

Address reprint requests to $R . J$. Bellon, Department of Radiology, MetroHealth Medical Center, 3395 Scranton Rd, Cleveland, $\mathrm{OH} 44109$.

Copyright $\odot 1992$ by W.B. Saunders Company 0897-1889/92/0501-0007\$03.00/0

\section{METHODS}

Serial CT scans of a formalin-preserved cadaveric foot and ankle were obtained. A Picker 1200 CT scanner (Picker International, Highland Heights, $\mathrm{OH}$ ) was used to acquire nonoverlapped $2-\mathrm{mm}$ thick slices, generating approximately 80 slices for an axial scan and 120 slices for a coronal* scan. Scans were performed at $130 \mathrm{kV}, 260$ milliAmpseconds. The slice data were transferred to a Reality Imaging Voxel Flinger (VF) (Reality Imaging, Solon, OH), a dedicated 3D image processing workstation. Using a surface rendering algorithm, the VF assembled these serial slices into 3D reconstructions of the foot.

\section{RESULTS}

Manipulations of the 3D reconstructions were successful in permitting differentiation of tendon from surrounding soft tissue. This was accomplished primarily through the VF thresholding ranges, which can be set so as to display any range of tissue densities. The VF can apply three independent density ranges simultaneously to the same volume data set. A single range can be adjusted to remove progressive amounts of lower density soft tissue (Fig 1). Addition of a second discrete density range corresponding to tendon allows their simultaneous viewing with bone.

Use of free-rotation along with thresholding allows each major tendon to be displayed along its entire course through the foot and ankle (Figs 2 and 3). In addition, 3D sections of the volume can be removed through a "cut" feature of the VF, thus permitting viewing of interior structures (Fig 3).

\section{DISCUSSION}

The VF has thresholding capabilities that allow the user to selectively visualize specific tissue densities (Fig 1). Certain tissues, for instance bone in the case of a CT scan, occupy a unique range in this density-space. They therefore lend themselves to discrete thresholding

\footnotetext{
${ }^{*}$ Axial and coronal are used here to refer to the anatomic position of the entire body; thus coronal sections are perpendicular to the long axis of the foot.
} 

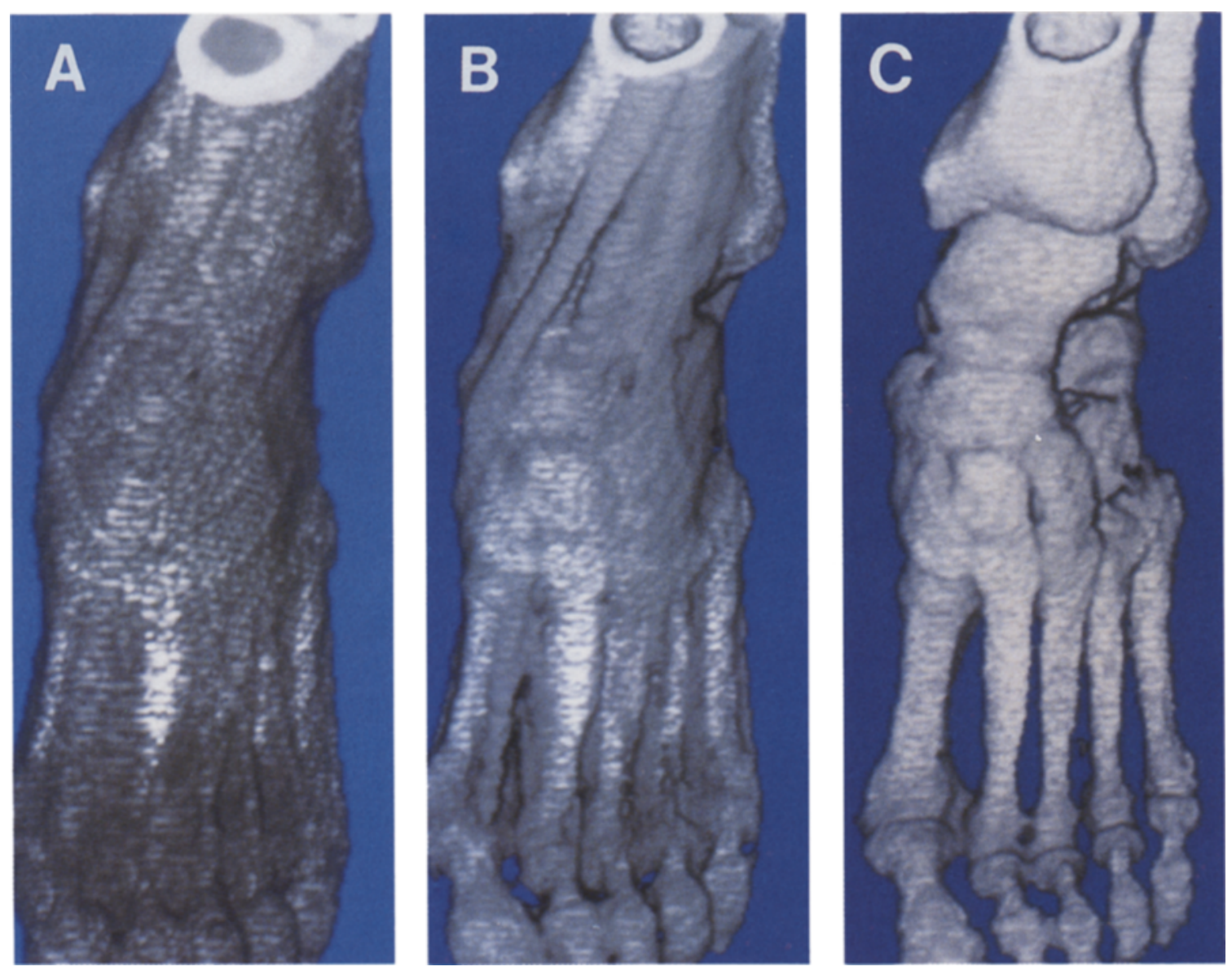

Fig 1. A 3D reconstructed image of an (undissected) cadaveric foot from serial axial CT slices, viewed from the dorsal aspect. Thresholding ranges are set in (A) so as to display the full range of tissue densities. Lower density soft tissue is increasingly removed (B), eventually leaving only bone (C).

and visualization. In CT, however, soft tissues are poorly segregated due to similarity in densities and partial volume effects; thus though it is possible to threshold the image data to display only bones, one cannot similarly isolate fat or muscle tissues.

Tendon densities, though not confined to as discrete a range as bone, did prove unique enough to enable visual isolation. We therefore chose to focus our study on tendons of the foot and ankle. Their densities lie slightly below those of bone, with the lower range overlapping slightly with muscle and skin (especially the callused areas of skin near the heel and ball of the foot).

We found the most informative views of the foot to be those with two active thresholding ranges: one displaying the bony framework, the other the tendons. The VF is also capable of real-time rotation about any axis, allowing the tendons to be easily traced from origin to insertion. This is particularly helpful for an understanding of the complex spatial relationships among the tendons of the plantar surface of the foot. For example, rendered images of the flexor digitorum longus and flexor hallucis longus tendons (Fig 3) clearly illustrate their crossing.

The VF can perform a limited number of cuts on a rendered image, removing a section of the image so as to expose structures otherwise hidden from view. Adler et $\mathrm{al}^{7}$ have used 3D techniques to disarticulate the boney structures of the ankle and foot and to remove overlying skin. Such computer manipulation is helpful when structures lie relatively far apart-eg, removing the tendo calcaneus so as to better visualize the path of the flexor hallucis longus 

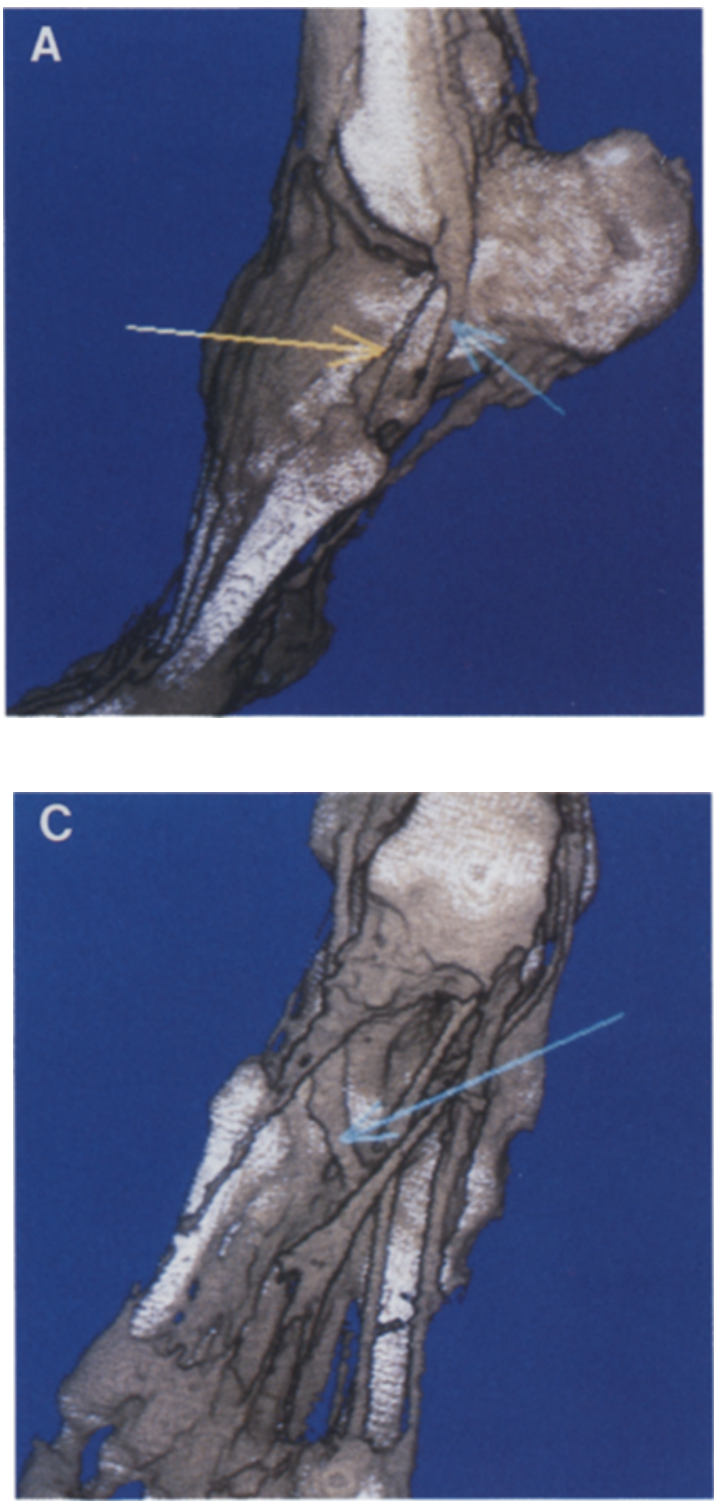

tendon along the posterior aspect of the ankle (Fig 3). However, the VF has limits in both the precision and the number of cuts. This makes it extremely difficult to computer-dissect away intricate structures. For example, cutting away the layers of the plantar surface in this manner proved impractical. It was primarily for this reason-to display the deep plantar structuresthat we dissected the foot manually and rescanned it. Figures 2 and 3 are from this re-scan, with skin and fascia removed from the foot. Figure 1 is a scan of the intact foot.

We are encouraged by the potential of 3D tendon imaging for education, where interactive manipulation permits clear 3D visualization and

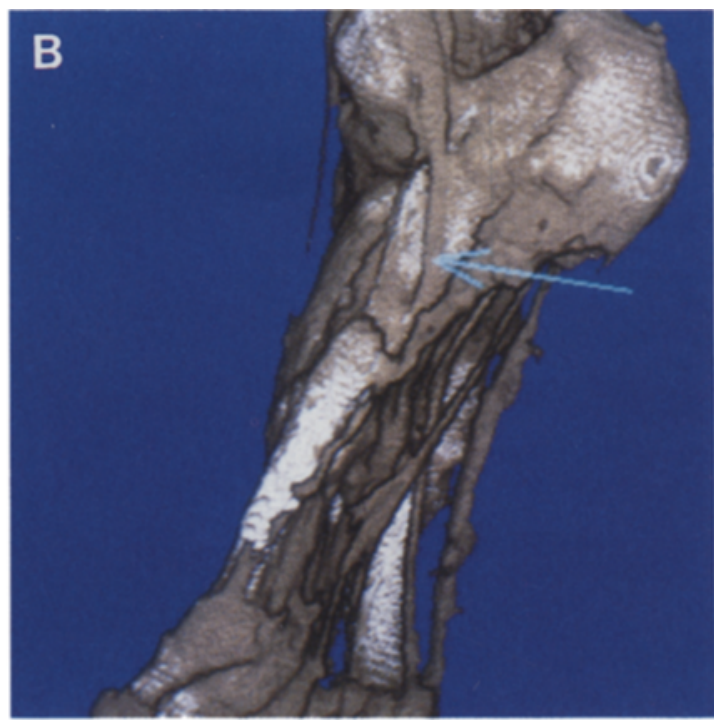

Fig 2. Scans of the foot with skin and fascia removed. The peroneus brevis (yellow arrow) and peroneus longus (blue arrow) tendons. The foot is rotated from a lateral view (A) through an oblique (B) to a plantar view (C). Note that the two tendons run together in a common sheath for a time. In regard to the peroneus brevis, note the insertion at the base of the first metatarsal. For the peroneus longus, note its passage across the sole of the foot to its insertion on the base of the first metatarsal and the medial cuneiform.

thus simplifies and reinforces the learning process. Three dimensional tendon reconstruction may have potential clinical utility as a noninvasive technique that permits viewing of entire tendons from any aspect. At the present time, however, clinical applications for diagnosis of tendon injury remain unassessed and require further study.

\section{ACKNOWLEDGMENT}

The authors acknowledge the assistance of the following people who contributed to this work: Errol Bellon MD, Alan Cohen MD, Jeff Crass MD, Debbie Cross-Blancke, Pedro Diaz PhD, Laura Hickel RT, Ed Kubiez RT, and Michael Pettit RT. 

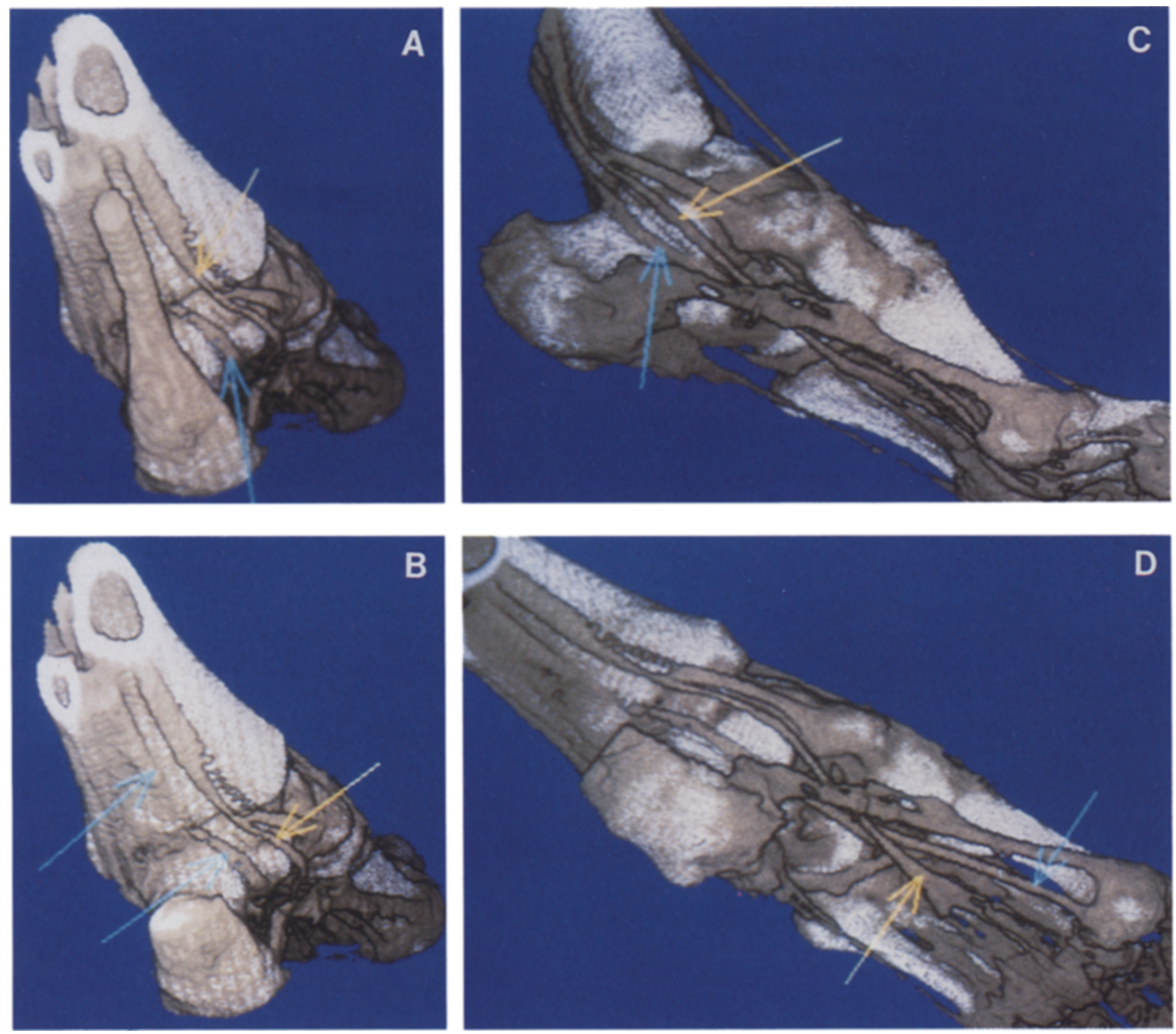

Fig 3. Scans of the foot with skin and fascia removed. The flexor digitorum longus tendon (Yellow arrow) and the flexor hallucis longus tendon (blue arrow). Note the path of the flexor digitorum longus tendon as it runs posterior to the medial malleolus (B), and its division into four smaller tendons inserting on the distal phalanges of the lateral four toes (D). These smaller divisions are not as well visualized due to "burning out" and partial voluming effects. In regard to the flexor hallucis longus tendon, note its passage through the groove created by the sustentaculum tali (C), its path deep to the flexor digitorum longus and between the sesamoid bones, and its insertion on the distal phalanx of the great toe $(D)$. It is partially obscured by the tendo calcaneus $(A)$ but is readily apparent on the cutaway views (B, C, and D).

\section{REFERENCES}

1. Robb WL: Future advances and directions in imaging research. AJR 150:39-42, 1988

2. Burk DL Jr, Mears DC, Kennedy WH, et al: Threedimensional computed tomography of acetabular fractures. Radiology 155:183-186, 1985

3. Fishman EK, Drebin RA, Magid D, et al: Volumetric rendering techniques: Applications for three-dimensional imaging of the hip. Radiology 163:737-738, 1987

4. Vannier MW, Marsh JL, Warren JO: Three-dimen- sional computer graphics for craniofacial surgical planning and evaluation. Comput Graph 17:263-273, 1983

5. Ney DR, Kuhlman JE, Hruban RH, et al: Threedimensional CT-volumetric reconstruction and display of the bronchial tree. Invest Radiol 25:736-742, 1990

6. Stern RL, Cline HE, Johnson GA, et al: Threedimensional imaging of the thoracic cavity. Invest Radiol 24:282-288, 1989

7. Adler SJ, Vannier MW, Gilula LA, et al: Threedimensional computed tomography of the foot: Optimizing the image. Comput Med Imaging Graph 12:59-66, 1988 